\title{
Melt instabilities on semiconductor surfaces induced by laser radiation
}

\author{
A.Yu. Bonchik, B.J. Dacko, V.I. Demchuk, S.G. Kiyak, I.P. Palyvoda, A.F. Shnyr \\ Pidstryhach Institute for Applied Problems of Mechanics and Mathematics, \\ National Academy of Sciences of Ukraine, 3-b Naukova Str., 79053 Lviv, Ukraine \\ e-mail:surface@iapmm.lviv.ua
}

\begin{abstract}
Laser modifications of semiconductors are often realized by pulsed rapid melting and subsequent resolidification. Recently melt instabilities have been discovered in locally melted semiconductor surfaces. We determined that spontaneous segregation of uniform temperature stage of crystal lattice and charge carrier concentration can take place in electronhole plasma, generated by laser radiation. Besides, there exists a positive feedback between the temperature of crystal lattice and charge carrier concentration in the region of their fluctuation. It causes both amplification of original fluctuation of temperature and generation of quasi-periodic temperature fields of large amplitude in semiconductors. The nonuniform temperature fields determine the features of surface relief formation in a zone of laser radiation action.
\end{abstract}

Keywords: instability, semiconductors, laser, electron-hole plasma, temperature fields.

Paper received 15.06.00; revised manuscript received 10.07.00; accepted for publication 12.07.00.

\section{Introduction}

The processes of heating semiconductors by laser radiation are the subject of many active studies (see, e.g. [1]) due to recent progress in non-equilibrium method of planned change of structure and electrical-physical properties of materials: laser solid-phase doping and laser annealing of semiconductors [2,3]. The features of effect of nanosecond laser pulses of duration $10 \ldots 100 \mathrm{~ns}$ on materials are studied in details. The numerical modeling makes it possible to analyze the time dependence of temperature on the surface of laser radiation zone and, besides, on different depths from the sample surface in this time regime of laser operation [1]. As to the effect on materials of laser pulses of millisecond and second ranges, only very approximate methods to calculate temperature fields [1] exist for these time regimes of laser action. The methods do not consider some important physical processes and heating mechanisms that seem to be effective for the above time ranges of laser operation. The processes that take place in the pulsed laser radiation zones are essentially non-equilibrium, which causes appearance of new physical phenomena due to the semiconductor parameters instabilities and self-organization in the systems disturbed from the thermodynamic equilibrium. In par- ticular, in electron-hole plasma (EHP), generated in semiconductors under laser radiation action, a spontaneous segregation of uniform state of temperature of crystal lattice and charge carriers concentration takes place [4,5].

The essence of the paper lies in the experimental and theoretical study of such effect in semiconductors under laser millisecond and second pulses action.

\section{Mathematical model and physics of appearance of temperature and charge carries concentration instabilities under uniform heat- ing the semiconductor}

Consider the peculiarities of interaction between a light flux and semiconductors at laser pulses of duration $>10^{-4}$ $\mathrm{sec}$ and at radiation power densities $<10^{5} \mathrm{~W} / \mathrm{cm}^{2}$. With such parameters of laser pulses, the concentration of photoinducted charge carries in the semiconductor of Siand Ge- type does not exceed $10^{18} \mathrm{~cm}^{-3}$ and electron-hole plasma is nondegenerate. Since the relaxation time for the energy of inducted electron-hole pairs is equal approximately to $10^{-12} \mathrm{sec}$, the electron-hole plasma can be considered as thermalized with a crystal lattice, and both the heat conduction and heat capacity of electrons and 


\section{A.Yu. Bonchik et al.: Melt instabilities on semiconductor surfaces induced by ...}

holes can be neglected. Under such assumptions the equation of temperature distribution is of the form [5]

$$
c \rho \frac{\partial T}{\partial t}=\nabla(\kappa(T) \nabla T)+\left(\alpha_{F}+\frac{\hbar \omega-E_{g}}{\hbar \omega} \alpha_{L}\right) I+R E_{g}
$$

where: $c, \rho, \kappa$ are the specific heat capacity, density, and heat conduction of a crystal lattice, $\nabla$ is the del-operator, $\alpha_{F}, \alpha_{L}$ are the coefficients of fundamental absorption and absorption by free charge carriers, $E_{g}$ is the forbidden zone width, $R$ is the velocity of charge carries recombination, $\hbar \omega$ is the energy of laser radiation quantum. The first term in the right-hand part corresponds to the temperature redistribution due to the heat conduction; the second one - to that part of energy which enters the crystal lattice due to electrons and holes thermalization at their constant concentration; the last term - to the heat transfer due to thermal recombination.

As a rule, while analyzing the pulse heating of the crystal lattice the electrons and holes concentration distribution is assumed to be given. In addition, the explicit dependence of the source in Eq.(1) on the generated carriers concentration is not essential. The situation is quite different when quasi-neutral electron-hole plasma can (due to the instability) redistribute on some bunches, what will cause essential change of absorption coefficients. The possibility of appearance of such instabilities was discussed in [6-8]. Utilizing the approach suggested in [6] we shall show that the instability of uniform pulse heating can be due to both thermodiffusion and bending of zones in the region of temperature fluctuations [6]. It will take place under more weaker conditions than in [68]. To study the processes of redistribution of charge carriers concentration we shall write the equations for electron-hole balance[5]:

$$
\frac{\partial n}{\partial t}=\nabla\left[D\left(\nabla n+\frac{n}{2 T} \nabla E_{g}+\frac{n}{T}(\alpha+1) \nabla T\right)\right]-R+G
$$

where $D$ is the coefficient of ambipolar diffusion, $G=\frac{\alpha_{L} I}{\hbar \omega}$ is the velocity of generating the charge carriers, $I$ is the light flux intensity, $R=\frac{n-n_{0}}{\tau_{r}}, \tau_{r}$ is the time of their recombination, $n_{0}$ is the balance charge carriers concentration. The first term in the right-hand part of Eq.(2) corresponds to diffusion redistribution that arises due to the concentration gradient with coefficient of ambipolar diffusion $D$, the second one corresponds to the ambipolar drift of electrons and holes in the electric field caused by local narrowing of the forbidden zone width [6]; the third one corresponds to the temperature redistribution due to thermodiffusion $[6,7]$.

For simplicity, consider the volume-uniform heating of semiconductor, when its thickness is less than the thickness of absorption of electromagnetic radiation $h<\alpha_{F}^{-1}, \alpha_{L}^{-1}$. To this end we assume that $\hbar \omega \approx E_{g}$. As a result, the source of Eq.(1) depends explicitly on the charge carriers concentration, and in such semiconductor the conditions of instability under uniform heating lattice can be realized. If one knowns self-consistent changes of $E_{g}$ with $n$ and $T$ under corresponding boundary and initial conditions, the particular temperature fields distribution in semiconductors can be found.

Consider the conditions for spontaneous formation of dissipative structures in semiconductors, caused by segregation of uniform state of charge carriers concentration and temperature of crystal lattice under pulse light excitation. To study this process Eqs (1),(2) are to be written in the following form

$$
\begin{aligned}
& c \rho \frac{\partial \Theta}{\partial t}=\nabla(\chi \nabla \Theta)+q(n, \Theta) \\
& \frac{\partial n}{\partial t}=\nabla\left[\frac{D_{0}}{\Theta^{\beta}} \nabla\left(n \Theta^{\alpha+1+\beta}\right)\right]+Q(n, \Theta)
\end{aligned}
$$

where $D=D_{0} \Theta^{\alpha+1} ; \Theta=\frac{T}{T_{0}} ; T_{0}$ is the initial temperature, $\beta=\frac{d \ln E_{g}}{d \ln T}, q, Q$ are the corresponding sources. The rest of parameters have the same values as in Eqs (1), (2).

Considering, that the charge carriers, excited by the light, dissipate their energy rapidly on the optical and acoustic phonons and uniformly generate in volume, the power $W$ is mainly spent on heating the semiconductor lattice. Assume also that the sample is heat-insulated, and the volume dissipation of energy during the heating time can be neglected. In this case the sources of Eqs(3),(4) can be given in the form

$q=\frac{1}{T_{0}}\left(\alpha_{F} I+E_{g} \frac{n}{\tau_{r}}\right), \quad Q=G-\frac{n}{\tau_{r}}$.

Assume that the concentration of non-equilibrium charge carriers exceeds considerably their balanced concentration. In addition, uniform heating of semiconductor lattice can be found from Eq. (4) when

$$
\frac{\partial \Theta}{\partial t}=\frac{q(n, \Theta)}{c \rho}
$$

The solution Eq. (6) can be found easily for different dependencies of the source $q$ on temperature:

$T_{h}=T_{0}+\int_{0}^{t} \frac{q\left(n_{0}, \Theta\right)}{c \rho} d t$. So, if $q$ does not depend on the temperature, the velocity of lattice temperature change from the heating time is given by a linear law

$T_{h}=T_{0}+\frac{q\left(n_{0}, T_{0}\right)}{c \rho} t$ 


\section{A.Yu. Bonchik et al.: Melt instabilities on semiconductor surfaces induced by ...}

By linearizing the Eqs (3),(4) in the vicinity of solution $T_{h}, n_{0}$ relative to the nonuniform disturbances

$\delta n, \delta \Theta \sim \exp \left(-\int_{0}^{t} \gamma\left(t^{\prime}\right) d t^{\prime}+\overrightarrow{i k} \vec{k}\right)$

we obtain the following dispersion equation:

$\gamma^{2} \tau_{r}^{2} \varepsilon-\gamma \tau_{r}\left(k^{2} L^{2}+k^{2} l^{2}+r-\xi \varepsilon^{-1}\right)+k^{4} L^{2} l^{2} \varepsilon+$

$+\zeta(\alpha+1+\beta) k^{2} L^{2}+r k^{2} l^{2} \varepsilon-\xi k^{2} L^{2}-\xi r-\zeta(s+g)=0$

where

$L^{2}=D_{0} \tau_{r} \Theta^{\alpha+1+s}, l^{2}=\chi \tau_{r} \Theta^{s}, \chi=\kappa \frac{1}{c \rho}$,

$\xi=\frac{d \ln \alpha_{F}}{d \ln \Theta}, \zeta=\frac{d \ln \alpha_{F}}{d \ln n}, 1-r=\frac{d \ln \tau_{r}}{d \ln n}$,

$s=\frac{d \ln \tau_{r}}{d \ln \Theta}, \varepsilon=\frac{T}{\tau_{r}}\left(\frac{d T}{d t}\right)^{-1}, \Theta=\frac{T}{T_{0}}, g=\frac{d \ln G}{d \ln \Theta}$,

$k^{2}=\left(\frac{\pi i}{l_{x}}\right)^{2}+\left(\frac{\pi j}{l_{y}}\right)^{2}+\left(\frac{\pi m}{l_{z}}\right)^{2}, l_{x}, l_{y}, l_{z}$

are the linear sizes of the sample, $i, j, m=1,2,3 \ldots m$.

As it follows from the dispersion equations, at satisfying the conditions $\xi r+\zeta(s+g)>0$, the growth increment $\gamma$ becomes less than zero for fluctuations from $k=0$, what corresponds to increase of absorption coefficient, i.e. to increase of velocity of uniform heating. However, the largest growth increment has the nonuniform fluctuations with a wave number

$k_{0}=\left[\xi-(\alpha+1+\beta) \zeta-\varepsilon r \frac{l^{2}}{L^{2}}\right]^{\frac{1}{2}}\left(2 \varepsilon l^{2}\right)^{-\frac{1}{2}}$

In addition, the velocity of critical heating depends essentially on the value $k_{0}$

$$
\frac{d \Theta}{d t}=\frac{\Theta}{\tau_{r}}\left(2 k_{0}^{2} l^{2}+r \frac{l^{2}}{L^{2}}\right)(\xi-\zeta(\alpha+1+\beta))^{-1}
$$

Then growth increment that corresponds to the nonuniform disturbances of temperature and concentration of EHP according to the value order is equal to $\frac{k_{0}^{2} L^{2}}{\tau_{r}}$.

The mechanism of instability considered is caused by dependence of the coefficient of light flux absorption on the charge carriers concentration and temperature, and also by thermodiffusion and dependence of the charge carriers flux on the change of forbidden zone width. Un- der fluctuation increase of charge carriers concentration in some region, the power of absorption of $\operatorname{EHP}(\zeta>0)$ increases and, by the same token, the local semiconductor heating increases. At high temperatures in not polar semiconductors, the charge carriers, in the main, scatter their pulse on the deformation potential of acoustic and optical phonons. Therefore, the diffusion coefficient decreases when the temperature $d \ln D / d \ln \Theta<0$ increases, and, hence, in the region of increased temperature of crystal lattice the thermodiffusion flux of charge carriers is also directed, that causes the subsequent increase of absorption degree of the light flux power. If the width of forbidden zone $E_{g}$ is a decaying temperature function, then $\beta<0$, and in the region of increased temperature the charge carriers flux will be also directed, which is caused by a local bending of zones. Therefore, the temperature distribution in the process of heating will be essentially nonuniform only when the velocity of leveling the nonuniform temperature distribution is less than that of the semiconductor heating.

Thus, the mechanism of instability of EHP under intensive light flux action on the semiconductors is caused, first of all by thermodiffusion of electrons and holes and by increase of temperature because of recombination in the regions with increased charge carriers concentration. In other words, the thermodiffusion instability of EHP is caused by pumping up (in the regions of higher temperature) the charge carriers additional concentrations which in turn, cause the increase of absorption degree of light flux and, by the same token, the temperature increase.

\section{Experimental results and conditions}

The nonuniform temperature fields arising in semiconductors under pulse laser radiation are determined experimentally. They define the specific features of materials melting on the initial stage of the process. Experiments are carried out on the samples of dislocation-free $\mathrm{Si}$, oriented in the planes (100), (110) and (111). Along with the samples prepared according to the generally accepted methods, the studies are carried out on the plates received by the method of the crystals spalling in vacuum in the device UP-5. The stay-time of a newly spalled surface in vacuum of order $4 \times 10^{-4} \mathrm{~Pa}$ does not exceed $1 \mathrm{sec}$. Besides, to reduce the thermal stresses arising in the zones of laser irradiation and leveling the temperature field, a thin $(\sim 200 \AA)$ amorphous film of $\mathrm{B}_{2} \mathrm{O}_{3}$ was deposited by vacuum evaporation on the crystal surface. The whole crystal surface was irradiated uniformly, where two types of lasers were used: continuous $\mathrm{CO}_{2}$ laser on $(\lambda=10.6 \mu \mathrm{m})$ of power $1 \mathrm{\kappa W}$ and pulse neodymium laser of GOS-300 $(\lambda=1.06 \mu \mathrm{m})$ type operating in the regime of free generation $\left(\tau_{1} \sim 10^{-3} \mathrm{sec}\right)$.

It is determined that independently of laser radiation wavelengths the material melting on the initial stage of the process is of local nature and takes place in separate discrete regions of the plate, where maxima of temperature field are situated. 

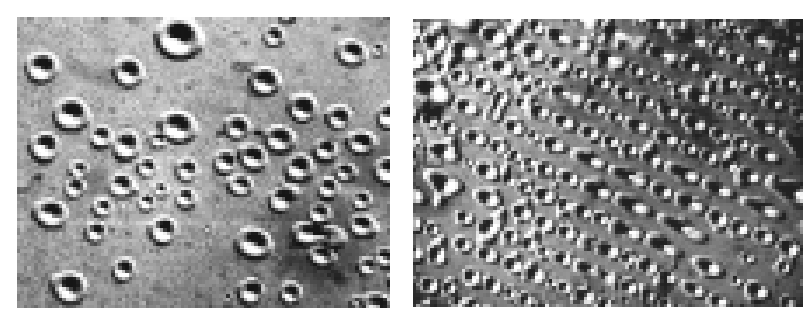

Fig. 1. Morphology of the surface of two-layer structure $\mathrm{B}_{2} \mathrm{O}_{3}-\mathrm{Si}$ in the zone of action of $\mathrm{CO}_{2}$ laser radiation. The density of light flux is: a) $-850 \mathrm{~W} / \mathrm{cm}^{2}$; b) $-900 \mathrm{~W} / \mathrm{cm}^{2}$

The studies of effect due to local melting of material surface in the zones of laser radiation action (without the influence of additional factors, to which in the first place thermal stresses and anisotropy of thermophysical characteristics of crystals are to be referred to) are carried out on the amorphous layer of $\mathrm{B}_{2} \mathrm{O}_{3}$, evaporated on the $\mathrm{Si}$ monocrystal surface.

At threshold densities of light flux energy on the surface of deposited layers the locally melted regions are formed; these, after crystallization, have a characteristic disk-shaped form (Fig. 1a). When the density of laser radiation energy is increased, the disk-shaped depressions of melts form a surface periodic structure (Fig. 1b), which verifies the fact of formation of the nonuniform quasi-periodic temperature fields with large amplitude in semiconductors at uniform laser excitation.
The morphology of surface of Si plates under anisotropy (natural and induced by thermal stresses) of thermophysical characteristics of crystals irradiation regions is shown in Fig. 2. Because of anisotropy of crystals properties the heat flux extends from the hot points in the directions of the largest heat conduction of crystal, which coincide with the diagonals of squares on the plane (100) and bisectors of angles of triangles on the plane (111) Fig. 2.

Thus, the nonuniform temperature fields arising in semiconductors under pulse laser irradiation of second and millisecond range, considering thermal stresses and anisotropy of thermophysical parameters of crystals, will induce the melt depressions, the form of which is connected definitely with crystallographic orientation of the semiconductor surface. E.g., on the plane (100) the square melt depressions are formed, on the plane(111) - triangular, and on the plane (110) - the hexagonal ones.

Moreover, local heating the surface of anisotropic semiconductors on the initial stage of melting can cause the induction of thermal stresses and strains, amplifying the anisotropy of thermal characteristics of crystals (Fig. 2a, b). It should be noted that when the density of light flux increases, the degree of effect of nonuniform heating the surface on the induced anisotropy of material characteristics decreases. This fact is due to, perhaps, increase of temperature in the surface layers on the whole. This causes: i) decrease of thermal stress level; and ii) decrease of the material heat conduction, which in its turn, induces the delay in the melt front motion along some crystallographic directions on the plate surface.

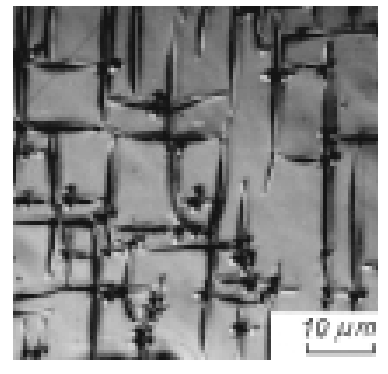

$\mathrm{a}$

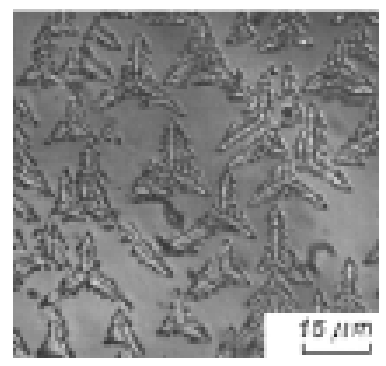

d

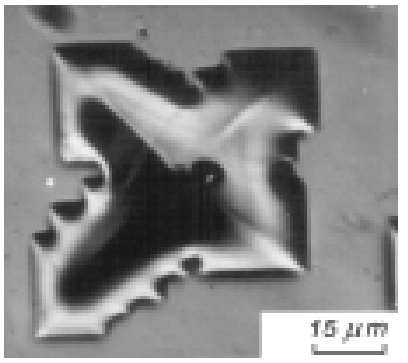

b

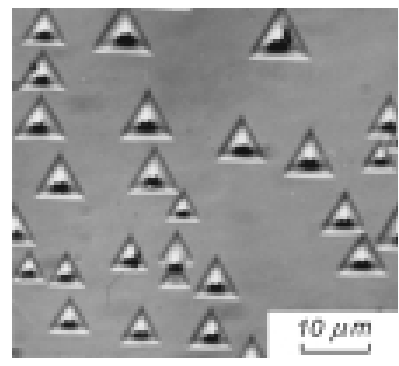

e

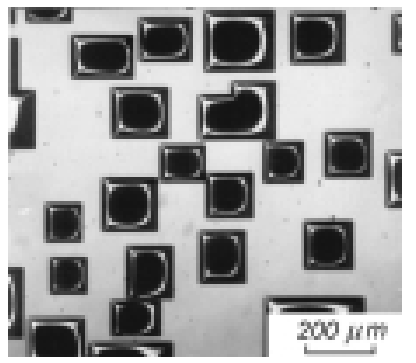

c

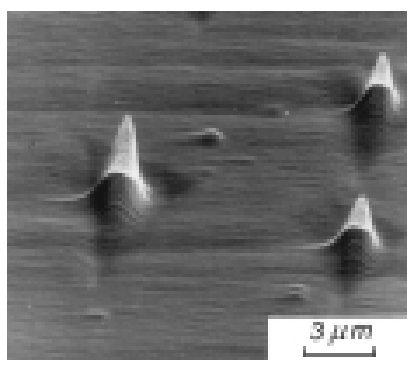

f

Fig. 2. Macropictures of Si surface in the zone of action of millisecond pulses of neodymium laser radiation (a,d,f) and second pulses of $\mathrm{CO}_{2}$ laser radiation on (b,c). g-relief of the surface (f) taken in the y-modulation regime. The surface orientation: (a-c) - (100), (d-g) - (111). 


\section{A.Yu. Bonchik et al.: Melt instabilities on semiconductor surfaces induced by ...}

The average distance between the local melts depressions under volume excitation is equal to $500 \mu \mathrm{m}$ (Fig.1), what is commensurable with the sample thickness and coincides well with calculated values of the period between the temperature field maxima. Considering that for Si $\beta \approx-2.5, \lambda+1 \approx-1, \mathrm{~s} \approx-1, \xi \approx 0 \div 1, \zeta=1, \tau_{r}=10^{-4}-10^{-5} \mathrm{~s}$ , $D=13 \mathrm{~cm}^{2} \mathrm{~s}^{-1}, \chi \approx 0.1 \mathrm{~cm}^{2} / \mathrm{s}$, we obtain estimates for characteristic length of the system $l \approx 10 \mu \mathrm{m}, L \approx 100 \mu \mathrm{m}$

and characteristic period of nonuniform temperature distributions $L_{x}=\frac{2 \pi}{k_{0}} \approx\left(T \chi\left(\frac{\partial T}{\partial t}\right)^{-1}\right)^{\frac{1}{2}}$. As it is seen, the most essential effect on the period between the melt depressions has the velocity of semiconductors heating, and for the velocity of heating $\frac{d T}{d t} \approx 10^{3} \div 10^{4} \frac{K}{s}$ it is equal to $\approx 500 \mu \mathrm{m}$.

Under millisecond pulses of neodymium laser action an average distance between the depressions of local melts decreases essentially due to higher heating velocities and larger values of absorption coefficients (Fig. 2). Thus, under millisecond pulses action the heating velocity increases in average by $10^{3}$ times, the characteristic period according to the above formula decreases by $10^{3 / 2}$ times, what is consistent with the experimental data.

\section{Conclusions}

The investigations show that in EHP, induced by pulse laser radiation, a spontaneous stratification of uniform temperature state of crystal lattice and charge carrier concentration can take place.

The nonuniform temperature fields define the peculiarities of melting processes, crystallization and semiconductor surface relief formation in the zones of light flux action. At uniform excitation of semiconductors by laser radiation with prethreshold power the locally melted regions are formed on the irradiated surface. The appearance of local melted regions is connected neither with interference effects, nor with dislocation structure of semiconductor. It is inducted by thermodiffusive instability of distribution of uniformly generated EHP, amplified by local strains and narrowing the width of semiconductor forbidden zone with temperature increase.

In addition, there exists a positive opposite connection between the temperature of a crystal lattice and charge carrier concentration in the region of their fluctuation. It causes both amplification of original fluctuations of temperature and generation of quasi-periodic temperature field of large amplitude.

\section{References}

1. Laser annealing of semiconductors Eds. J.M.Poate, James W.Mayer, Academic Press, New York, (1982).

2. A.M.Prokhorov, A.Yu.Bonchik, S.G.Kiyak, A.A.Manenkov, G.N.Mikhailova, A.V.Pokhmurskaja, A.S.Seferov, I.Ursu, V.Craciun, I.N.Mihailescu, Laser solid -phase doping of semiconductors // Appl. Surf. Sci., 43, pp.340-345, (1989).

3. A.Pokhmurska, O.Bonchik, S.Kiyak., G.Savitski, A.Gloskovski, Laser doping in Si, InP and GaAs // Appl. Surf. Sci., 712, pp.154-155 (2000)

4. A.Yu.Bonchik, V.V.Gafijchuk, S.G.Kiyak, G.V.Savitsky, Morphology of semiconductors surface under the action of laser pulses of millisecond duration // Poverkhnost' (fizika, chimiya, mechanika), 5, pp.1883-1888, (1986) (in Russian).

5. S.Kiyak, A.Bonchik, V.Gafijchuk, A.Yuzhanin, I.Tysliuk, A.Pokhmurskaja, Periodical structures formation on the surface of semiconductors under the laser irradiation // Izvestija AN USSR, 52(11), pp.2276-2281, (1988) (in Russian).

6. V.Gafijchuk. Temperature instability at uniform heating of semiconductors by pulse radiation // Phizika tverdogo tela, 26(7), pp.2230-2231 (1985) (in Russian).

7. V.V.Gafijchuk, V.E.Gashpar. Appearance of heterogeneous structures at pulse heating of semiconductors // Phizika tverdogo tela, 27(5), pp.1354-1358, (1985) (in Russian).

8. V.V.Gafijchuk, V.E.Gashpar. Quasi-periodic temperature fields in semiconductors at laser treatment.// DAN USSR, ser $A$, vyp 9, pp.74-78 (1985) (in Russian). 